\title{
SEISMic Response of AsymMetric Systems: ENERGY-BASED APPROACH
}

\author{
By Rakesh K. Goel
}

\begin{abstract}
ABSTAACT: An energy-based approach has been used to investigate the seismic behavior of code-designed asymmetric-plan systems. The systems considered in this investigation had large eccentricities in both directions, were designed for bidirectional earthquake loading, and their responses were computed with both components of ground motion acting simultaneously. The presented results demonstrate that the total input energy is about the same whether the system plan is symmetric or asymmetric. Furthermore, flexible-side elements in asymmetric-plan systems may be more vulnerable to earthquakes than the same elements in symmetric-plan systems if they are not designed to accommodate the higher hysteretic-energy demands. This observation correlates well with the damage observed in street comer buildings during several earthquakes. Stiff-side elements, on the other hand, are expected to suffer no more damage in asymmetric-plan systems than in symmetric-plan systems.
\end{abstract}

\section{INTRODUCTION}

It has been well recognized that asymmetric-plan buildings are especially vulnerable to earthquakes due to coupled lateral and torsional motions. The effects of such coupling and how well these effects are represented in seismic codes have been the subject of numerous investigations (Rutenberg 1992). A brief review of the literature shows that most investigations examined inelastic earthquake response of simple one-story, monosymmetric, asymmetric-plan systems subjected to either one component (Chopra and Goel 1991; De Stefano et al. 1993; Goel and Chopra 1990, 1994; Tso and Zhu 1992) or two components (Wong and Tso 1995) of ground motion. The effects of coupling between lateral and torsional motions in code-designed systems were generally evaluated by comparing element ductility demands in asymmetric-plan and the corresponding symmetric-plan (or reference) systems. These investigations generally concluded that elements on the stiff side (the same side of the center of mass as the center of rigidity) in code-designed, asymmetric-plan systems are likely to suffer more damage, whereas elements on the flexible side (the side opposite the stiff side) are expected to suffer less or similar damage compared to those in the reference system. The damage observed during the 1985 Mexico earthquake (Esteva 1987) and 1995 Kobe earthquake (Whittaker 1995), however, indicated otherwise. During these earthquakes many buildings located at the intersection of two streets suffered damage to their sides facing the streets. The frames of these corner buildings had large openings on the sides facing the streets, whereas the frames on sides bordering neighboring buildings were filled with masonry walls. The flexible-side elements in such buildings were located on the open sides, which were damaged significantly. This observation clearly contradicts the findings of the aforementioned analytical studies.

In a review of the reference models used in studies on effects of torsion in buildings responding in the inelastic range, Correnza et al. (1992) concluded that ignoring the accidental eccentricity in design of the reference system, as was the case in aforementioned investigations, leads to significant changes in its inelastic response. A series of the following investigations (Chandler et al. 1995; Correnza et al. 1995) showed that if accidental eccentricity is included in the design of the ref-

Asst. Prof., Dept. of Civ. and Envir. Engrg., Calif. Polytech. State Univ., San Luis Obispo, CA 93407. erence system, ductility demands on the flexible-side element of asymmetric-plan systems designed according to codes that do not amplify the static eccentricity may exceed the values in the reference system. Another recent investigation (Chandler et al. 1996) reached the same conclusion by examining the hysteretic-energy dissipation demands. Although the findings of these studies appear to correlate with the observations during the 1985 Mexico and 1995 Kobe (Japan) earthquakes, they were also based on inelastic responses of one-story, monosymmetric structural systems subjected to only one component of the ground motion. It will be useful to reexamine and verify these findings using structural systems similar to the street corner buildings, i.e., structural systems with large eccentricities in both directions, which were found to suffer significant damage during recent earthquakes.

With the objective of filling this need, this investigation was focused on earthquake behavior of one-story asymmetric-plan systems - with large eccentricities in both directions and designed for bidirectional earthquake loading - simultaneously subjected to both components of the ground motion. In particular, this study investigated how various energy quantities differ between the code-designed asymmetric- and symmetricplan systems. For this purpose, the ways in which torsional provisions in building codes influence the element and total design forces were examined first. Next, force-deformation histories and energy spectra of asymmetric- and symmetricplan systems designed according to one of the major seismic codes were compared to understand how plan asymmetry influences energy quantities. Finally, energy-based and ductilitybased indices of asymmetric-plan systems designed according to three major seismic codes were compared with symmetricplan systems.

\section{SYSTEM AND GROUND MOTIONS}

\section{One-Story System}

The system considered was the idealized one-story building of Fig. 1. This system consisted of a rigid deck supported by three structural elements in each of the two orthogonal directions. The structural elements were frames or walls having strength and stiffness in their planes only. The mass properties of the system were assumed to be symmetric about both the $X$ - and $Y$-axes. As a result, the center of mass (CM) of the system coincided with its geometric center. The stiffness properties of the system were, however, not symmetric about the geometric center. This lack of symmetry was characterized by the stiffness eccentricities, $e_{s x}$ and $e_{s y}$, defined as $X$ - and $Y$ components of the distance between the CM and the center of rigidity $(\mathrm{CR})$, respectively.

The linearly elastic responses-displacements in the $X$ - and 


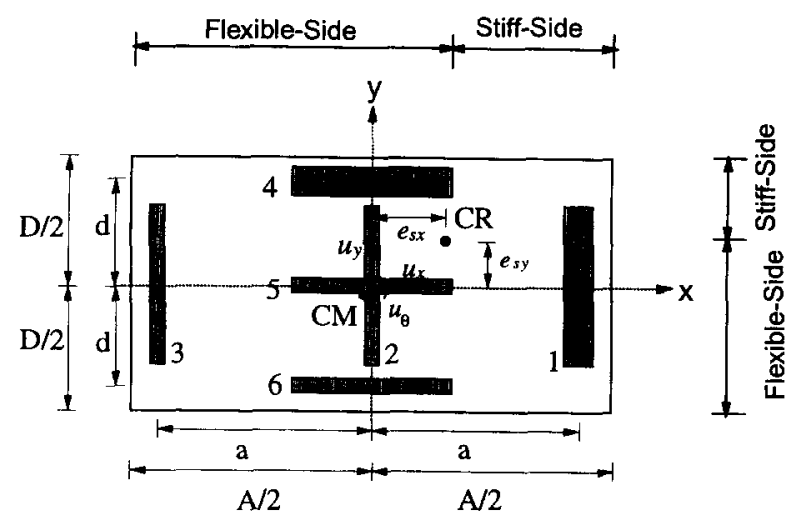

FIG. 1. Idealized One-Story System

$Y$-directions, $u_{x}$ and $u_{y}$, and rotation about the vertical axis, $u_{\theta}$ - of such a system depend on the following parameters: uncoupled translational period in the $Y$-direction, $T_{y}\left(=2 \pi / \omega_{y}\right)$; ratio of uncoupled translational frequencies in the $X$ - and $Y$ directions, $\Omega_{x}=\omega_{x} / \omega_{y}\left(=T_{y} / T_{x}\right)$; uncoupled torsional to translational frequency ratio, $\Omega_{\theta}=\omega_{\theta} / \omega_{y}\left(=T_{y} / T_{\theta}\right)$; normalized stiffness eccentricities in the $X$ - and $Y$-directions, $\bar{e}_{s x}=e_{s x} / A$ and $\bar{e}_{s y}=e_{s y} / D$; aspect ratio, $\eta=A / D$; and damping ratio, $\xi$, in each mode of vibration. The aspect ratio was included as one of the system parameters because it facilitated a more appealing definition of the stiffness eccentricity as a percentage (or fraction) of the plan dimension. If the stiffness eccentricity was normalized by the mass radius of gyration, the aspect ratio would not be an independent system parameter.

The nonlinear responses depend not only on the elastic system parameters but also on the distribution of strength and stiffness in the plan of the system. Therefore, it was necessary to specify the location and strength of each resisting element. The central elements in each direction (denoted as 2 and 5) were selected to be located at the CM of the system plan. The outer elements (numbered 1, 3, 4, and 6) were selected to be located symmetrically about the CM. The elements oriented along the $Y$-direction (denoted as 1 and 3 ) were located at a distance $a(\leq 0.5 A)$, whereas those oriented along the $X$-direction (denoted as 4 and 6) were located at a distance $d(\leq 0.5 D)$ from the CM. These locations were determined from the following relationships:

$$
\begin{gathered}
\bar{a}=\frac{a}{A}=0.25\left[-\bar{e}_{s x} \pm \sqrt{25 \bar{e}_{s x}^{2}+2\left(1-\gamma_{x}\right) \Omega_{\theta}^{2}\left(1+\eta^{2}\right) / \eta^{2}}\right] \\
\bar{d}=\frac{d}{D}=0.25\left[-\bar{e}_{s y} \pm \sqrt{25 \bar{e}_{s y}^{2}+2 \gamma_{x} \Omega_{\theta}^{2} \Omega_{x}^{2}\left(1+\eta^{2}\right)}\right]
\end{gathered}
$$

in which an additional parameter, $\gamma_{x}$, was used. This parameter was defined as the ratio of the torsional stiffness provided by the $X$-direction elements to the total torsional stiffness of the system. Eqs. (1) and (2) indicate that locations of outermost elements are not fixed (they vary in the building plan, depending on various parameters involved in these equations). Several previous studies [e.g., Correnza et al. (1992); Wong and Tso (1995)] assumed the outer elements to be located at the edges of the system plan; fixing these locations implies additional restrictions and will make it physically impossible to independently vary both $\gamma_{x}$ and $\Omega_{x}$.

Once locations of all the elements were determined, their stiffnesses, normalized by the system's mass $m$, were computed as

$$
\begin{aligned}
& \frac{k_{y 1}}{m}=\frac{1}{3} \omega_{y}^{2}\left[1+2 \frac{\bar{e}_{s x}}{\bar{a}}\right] \text { and } \frac{k_{x 2}}{m}=\frac{k_{y 3}}{m}=\frac{1}{3} \omega_{y}^{2}\left[1-\frac{\bar{e}_{s x}}{\bar{a}}\right] \\
& \frac{k_{x 4}}{m}=\frac{1}{3} \omega_{y}^{2} \Omega_{x}^{2}\left[1+2 \frac{\bar{e}_{s y}}{\bar{d}}\right] \text { and } \frac{k_{x 5}}{m}=\frac{k_{x b}}{m}=\frac{1}{3} \omega_{y}^{2} \Omega_{x}^{2}\left[1-\frac{\bar{e}_{s y}}{\bar{d}}\right]
\end{aligned}
$$

It is useful to state that not all the parameters used in the formulation of the system model are entirely independent. In particular, the values of some of these parameters are constrained by the physical restriction that locations $a$ and $d$ of the outer elements should be within the building plan. In this investigation, selected values of the parameters satisfied these constraints.

\section{Ground Motions}

The following five pairs of earthquake records were considered as input ground motion for the inelastic response analysis: (1) Castaic Old Ridge Rte., 1994 Northridge earthquake; (2) Pacoima Kagel, 1994 Northridge earthquake; (3) Castaic Old Ridge Rte., 1971 San Fernando earthquake; (4) Taft Lincoln School Tunnel, 1952 Kern County earthquake; and (5) El Centro, 1940 Imperial Valley earthquake. These records are all from rock sites in California and were selected because their elastic response spectra are similar to each other and to the Newmark-Hall design spectrum. All the records were scaled to $0.4 \mathrm{~g}$ peak acceleration, which was also the peak acceleration used for the system design. The larger of the two components of each earthquake (prior to scaling) was assumed to act in the $Y$-direction and the other in the $X$-direction.

\section{DESIGN OF ASYMMETRIC-PLAN SYSTEM}

\section{Lateral Design Force}

The design forces in the $X$ - and $Y$-directions were calculated

$$
F_{x}=\frac{m A_{x}}{R_{x}} ; \quad F_{y}=\frac{m A_{y}}{R_{y}}
$$

in which $R_{x}$ and $R_{y}=$ reduction factors in the $X$ - and $Y$-directions, respectively; and $A_{x}$ and $A_{y}=$ pseudoaccelerations selected from the $5 \%$ damped Newmark-Hall design spectrum constructed with peak values of ground acceleration $=0.4 \mathrm{~g}$, velocity $=36.5 \mathrm{~cm} / \mathrm{s}(14.37 \mathrm{in} . / \mathrm{s})$, and displacement $=10 \mathrm{~cm}$ (3.75 in.). The reduction factors depend on the capacity of the system to safely undergo inelastic deformation during intense ground shaking. For the selected values of $R_{x}=R_{y}=4$, the system was expected to be excited well into the inelastic range during the earthquakes considered in this study.

\section{Design Eccentricity}

Most seismic codes require that the lateral earthquake force at each floor level of an asymmetric-plane building be applied at a distance equal to the design eccentricity from the CR. The design eccentricity specified in most seismic codes is of the form (Goel and Chopra 1990)

$$
\begin{aligned}
& e_{d}=\alpha e_{s}+\beta b \\
& e_{d}=\delta e_{s}-\beta b
\end{aligned}
$$

where $b=$ the plan dimension of the building perpendicular to the direction under consideration $(=B$ or $D$ for design in the $Y$ - or $X$-direction); $e_{s}=$ the eccentricity between the $\mathrm{CM}$ and $\mathrm{CR}\left(=e_{s x}\right.$ or $e_{s y}$ for design in the $Y$ - or $X$-direction); and $\alpha, \beta$, and $\delta=$ code-specified coefficients: $\alpha=\delta=1$ and $\beta=0.05$ in UBC-94 (Uniform 1994); $\alpha=1.5, \delta=0.5$, and $\beta=0.1$ in NBCC-95 (National 1995); and $\alpha=1.5, \delta=1$, and $\beta=0.1$ in MFDC-87 (Gomez and Garcia-Ranz 1988). For each element the design eccentricity value leading to the larger design force is used. The first term in these equations is intended to account for the lack of symmetry in the building plan, whereas the second term, denoted as the accidental eccentricity, is included to consider torsional effects due to factors not explicitly considered. 
UBC-94 (Uniform 1994) also specifies an amplification factor for the accidental eccentricity given as

$$
A_{x}=\left(\frac{\delta_{\max }}{1.2 \delta_{\text {avg }}}\right)^{2} ; 1 \leq A_{x} \leq 3
$$

in which $\delta_{\max }$ and $\delta_{\mathrm{avg}}$ are the maximum and average displacements of the floor diaphragm, respectively, when the structure is subjected to the code-defined static lateral loading. This amplification was included in designing the asymmetric-plan system according to the torsional provision of UBC-94.

\section{Element Design Forces}

The element design forces were computed in two steps. The first step involved computation of the element forces due to independent application of $F_{x}$ and $F_{y}$. Application of the lateral force in the $Y$-direction, $F_{y}$, at a distance equal to the design eccentricity, $e_{d x}$, from the $\mathrm{CR}$ led to the following element forces:

$$
\begin{gathered}
f_{i}^{y}=F_{y} \frac{k_{y i}}{K_{y}}\left[1+\frac{12 \eta^{2}}{1+\eta^{2}} \frac{1}{\Omega_{\theta}^{2}} \bar{e}_{d x}\left(-\bar{x}_{i}+\bar{e}_{x x}\right)\right] ; \quad i=1,2 \text {, and } 3 \\
f_{i}^{y}=F_{y} \frac{k_{x i}}{K_{y}}\left[\frac{12 \eta}{1+\eta^{2}} \frac{1}{\Omega_{\theta}^{2}} \bar{e}_{d x}\left(\bar{y}_{i}-\bar{e}_{s y}\right)\right] ; \quad i=4,5 \text {, and } 6
\end{gathered}
$$

in which $\bar{e}_{d x}=e_{d x} / A$ is the normalized design eccentricity in the $X$-direction; and $\bar{x}_{i}=x_{i} / A$ and $\bar{y}_{i}=y_{i} / D$ are the normalized distances of the $Y$ - and $X$-direction elements, respectively, from the CM. The design eccentricity, $e_{d x}$, was obtained by utilizing $e_{s}=e_{s x}$ in (6a) and (6b). Similarly, application of the lateral force in the $X$-direction, $F_{x}$, at a distance equal to the design eccentricity, $e_{d y}$, from the CR led to the following element forces:

$$
\begin{gathered}
f_{i}^{x}=F_{x} \frac{k_{y i}}{K_{x}}\left[\frac{12 \eta}{1+\eta^{2}} \frac{1}{\Omega_{\theta}^{2} \Omega_{x}^{2}} \bar{e}_{d y}\left(\bar{x}_{i}-\bar{e}_{s x}\right)\right] ; i=1,2 \text {, and } 3 \\
f_{i}^{x}=F_{x} \frac{k_{x i}}{K_{x}}\left[1+\frac{12}{1+\eta^{2}} \frac{1}{\Omega_{\theta}^{2} \Omega_{x}^{2}} \bar{e}_{d y}\left(-\bar{y}_{i}+\bar{e}_{s y}\right)\right] ; i=4,5 \text {, and } 6
\end{gathered}
$$

in which $\vec{e}_{d y}=e_{d y} / D$ is the normalized design eccentricity in the $Y$-direction, with $e_{d y}$ computed by using $e_{s}=e_{s y}$ in $(6 a)$ and $(6 b)$.

The term inside the square brackets in (8)-(11) may be interpreted as the element force in an asymmetric-plan system normalized by the force in the same element of a corresponding symmetric-plan system. The corresponding symmetricplan system, also denoted as the reference system in the rest of this paper, was defined as a system with coincidental $\mathrm{CM}$ and $C R$ but with relative locations and stiffnesses of all elements identical to those in the asymmetric-plan system. Therefore, the term inside the square brackets in these equations is indicative of a modification of element forces due to plan asymmetry.

The second step in computing the element design forces involved accounting for the orthogonal effects. In general, seismic codes specify that an element be designed for $100 \%$ of the force resulting from application of the seismic force in one direction, plus a certain percent (usually 30 or $40 \%$ ) of the force from application of the seismic force in the perpendicular direction. This investigation, however, used the following combination rule for including orthogonal effects (Wilson et al. 1995):

$$
f_{i}=\sqrt{\left(f_{i}^{x}\right)^{2}+\left(f_{i}^{y}\right)^{2}} ; \quad i=1-6
$$

Since UBC-94 does not permit reduction of element design forces due to torsion, the design force, $f_{i}$, in each element of a system designed according to this code was selected equal to at least the design force in the same element of the reference system.

The design forces in the extreme lateral-load resisting elements 1 and 3 oriented along the $Y$-direction (Fig. 1), normalized by the design force in the same elements of the reference system, are shown in Fig. 2 for the three codes. In remainder of this paper, element 1 , which is located on the stiff side (the same side of the CM as the CR), is denoted as the stiff-side element, and element 3 , which is located on the flexible side (the side opposite the stiff side), is identified as the flexible-side element. The design forces in the stiff-side and flexible-side elements oriented along the $X$-direction, i.e., elements 4 and 6 , are not included because they were the same as in elements 1 and 3, respectively, for the system considered in this investigation. In calculating design forces in elements of the reference system, the accidental eccentricity was ignored (Rutenberg 1992; Wong and Tso 1995). These results show that the normalized strength for a stiff-side element designed according to UBC-94 is either larger than or equal to 1. The values larger than 1 occur for small values of eccentricity for which the element strength is controlled by the accidental eccentricity. Since UBC-94 does not permit reduction

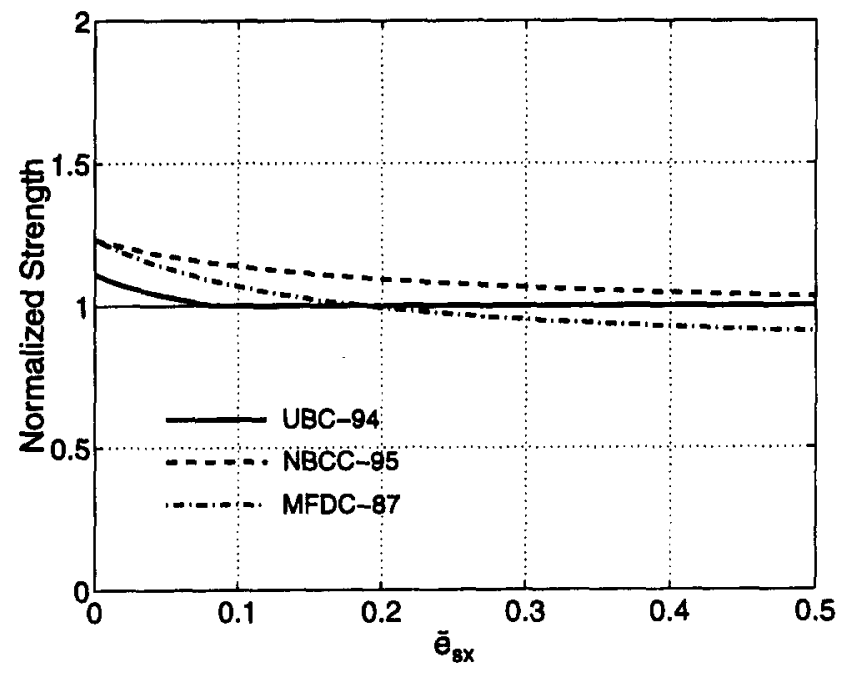

(a)

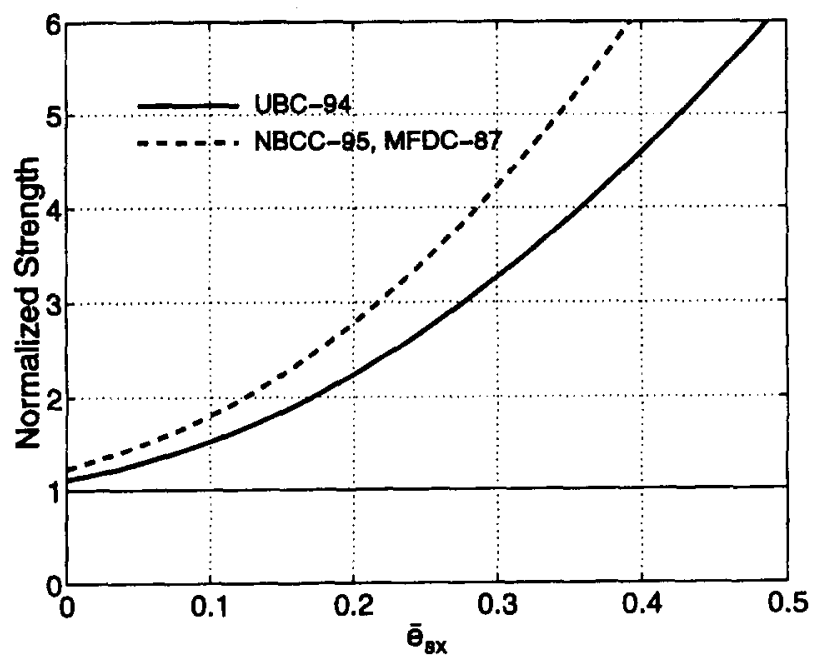

(b)

FIG. 2. Normalized Strength of Elements in Code-Designed Asymmetric Systems: $\delta_{e y}=\delta_{a x} \Omega_{\theta}=\Omega_{x}=\eta=1$, and $\gamma_{x}=0.5$ : (a) Stiff-Side Element; (b) Flexible-Side Element 
due to torsion, the normalized strength of the stiff-side element is equal to 1 for larger values of eccentricity. Normalized strength of this element in systems designed according to NBCC-95 is always larger than 1, even though this code permits a reduction in the element strength due to torsion. A detailed investigation revealed that normalized design force remained larger than 1 because of the orthogonal effects considered in this study. Since MFDC-87 permits larger reduction $(\delta=1)$, the normalized strength of the stiff-side element in systems designed according to this code is generally smaller compared to the NBCC-95 and may become smaller than 1 for very large values of eccentricity.

The normalized strength of the flexible-side element in systems designed according to all three codes is always larger than 1 , indicating that all codes require an increase in design strength of this element. Since the coefficient $\alpha$ in $(6 a)$, which controls the strength of this element, is larger for the NBCC95 and MFDC-87 $(\alpha=1.5)$ as compared to UBC-94 $(\alpha=1.0)$, the strength of this element is larger due to the former codes. Furthermore, NBCC-95 and MFDC-87 led to the same strength because these codes specify identical values of $\alpha=$ 1.5. The increase in the strength of the flexible-side element is particularly large for systems with large eccentricity. For example, the strength of this element in a system with $\bar{e}_{s x}=$ $\bar{e}_{s y}=0.3$ (30\% eccentricity in both directions) is about three times greater if designed according to UBC-94 and a little more than four times greater if designed according to NBCC95 and MFDC-87, the strength of the same element in the reference system. Such a large increase in strength of this element was not observed in previous investigations [e.g., Wong and Tso (1995); Chopra and Goel (1991)] where monosymmetric systems were studied. Obviously, the much larger increase in strength observed in the present study is due to the orthogonal effects in systems with large eccentricities in both directions.

\section{Overstrength Factor}

The overstrength factor, defined as the ratio of the total strength of all elements in the $Y$-direction of the asymmetric system and the reference system, is plotted in Fig. 3 against the normalized eccentricity, $\vec{e}_{s x}$. The overstrength factor for all codes is larger than 1, indicating that the total strength of the asymmetric-plan system is larger than that of the reference system. Values of the overstrength factors were the smallest from UBC-94 and largest from NBCC-95; MFDC-87 led to values between these extremes. For large values of eccentricity, the overstrength factor may be significant. For example,

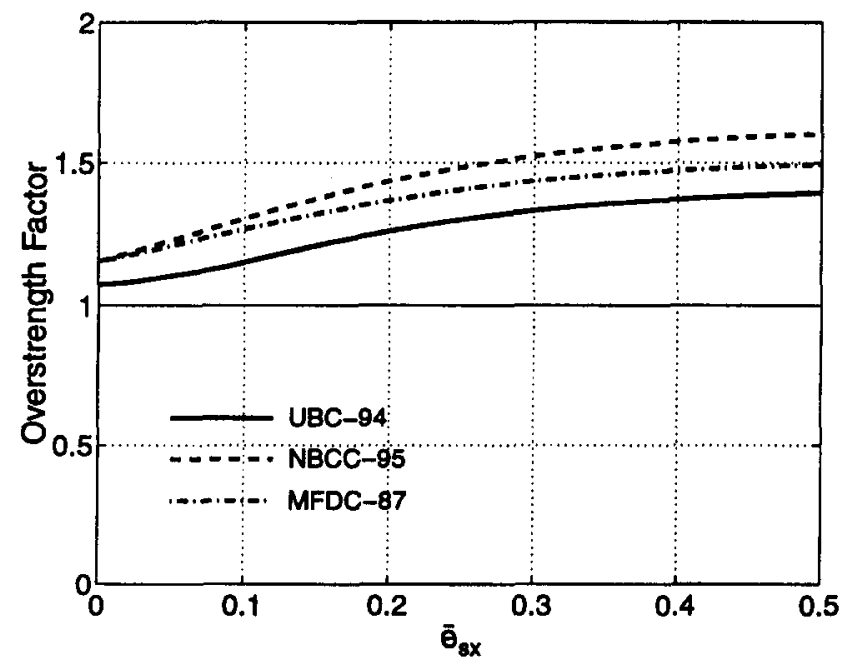

FIG. 3. Overstrength Factor in Code-Designed Asymmetric Systems: $\delta_{a y}=\delta_{a x} \Omega_{0}=\Omega_{x}=\eta=1$, and $\gamma_{x}=0.5$ the overstrength factor in a system with $\bar{e}_{s x}=\bar{e}_{s y}=0.3$ was about 1.3 if designed according to UBC-94 and a littler over 1.5 if designed according to NBCC-95. Such large overstrength factors are, as mentioned in the preceding section, due to the orthogonal effects.

\section{SYSTEM PARAMETERS AND RESPONSE QUANTITIES}

The inelastic responses of one-story asymmetric-plan systems and their reference systems, with both components of selected earthquakes acting simultaneously, were computed by step-by-step integration of the equations of motion. For this purpose the constant acceleration Newmark-Beta integration method with iteration was used. The damping matrix was defined as a linear combination of the mass matrix and the initial stiffness matrix, with the multipliers selected for $5 \%$ damping in each of the first two modes of vibration.

\section{Selected System Parameters}

The inelastic responses are presented for the following values of system parameters. Values of $T_{y}$ were selected in the range of $0.1-3 \mathrm{~s}$ to represent many low-rise and mid-rise buildings. The value of $\Omega_{\theta}$ was selected to be unity, for which significant coupling between lateral and torsional motions is expected in the elastic range. The selected value of $\Omega_{x}=1$ corresponds to identical uncoupled vibration periods in the two orthogonal directions, and $\gamma_{x}=0.5$ corresponds to an equal contribution to the system's torsional stiffness from the lateralload resisting elements oriented along the two directions. The normalized stiffness eccentricities in the two directions, $\bar{e}_{s x}$ and $\vec{e}_{s y}$, were selected to be the same and equal to 0.3 . The large eccentricities considered in this investigation are representative of the values of many street corner buildings that are composed of moment-resisting frames to accommodate windows on the street frontages and stiff infill masonry supported by moment-resisting frames or concrete walls on the remaining faces. The aspect ratio, $\eta$, of the selected systems were fixed at 1 .

The yield strengths of the resisting elements were computed according to the torsional provisions of the three major seismic codes using the procedure described in the preceding section. The force-deformation behavior of each resisting element was selected as elastoplastic with $3 \%$ postyield strain hardening.

\section{Inelastic Responses}

The primary response quantities considered in this investigation were the hysteretic energy dissipation demands on the stiff-side and flexible-side elements oriented along the $Y$-direction (elements 1 and 3 in Fig. 1). Since the trends are expected to be similar for the stiff-side and flexible-side elements oriented along the $X$-direction (elements 4 and 6 in Fig. 1), results for these elements are not included. For selected systems, total seismic input energy and energy dissipated through damping were also computed. Relative (and not absolute) seismic input energy formulation was used in this investigation because of its simplicity in implementation. Details of procedures to compute various energies are available elsewhere (Uang and Bertero 1990).

The hysteretic energy demand on the lateral-load resisting elements was further characterized by a factor similar to the displacement ductility demand. This factor, denoted as the normalized hysteretic energy ductility demand (NHEDD), was defined as one plus total hysteretic energy dissipated by the element during all inelastic cycles divided by twice the energy absorbed at the first yield (Mahin and Bertero 1981). NHEDD represents the maximum displacement ductility demand in an 


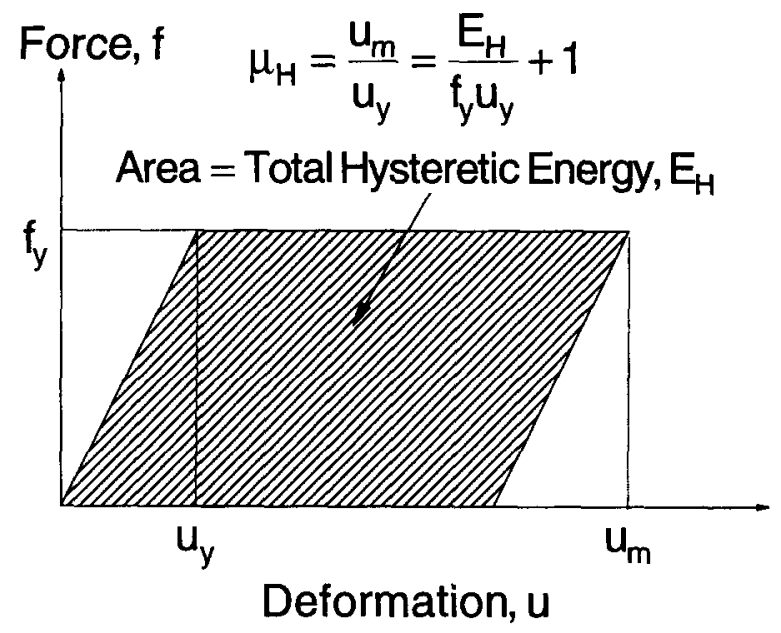

FIG. 4. Definition of Normalized Hysteretic Energy Ductility Demand [after Mahin and Bertero (1981)]

equivalent elastic perfectly plastic element that dissipates under monotonic loading the same amount of hysteretic energy as the actual element (Fig. 4). Results were also generated for the peak displacement ductility demand (PDDD) for comparison purposes and force-deformation histories of selected systems to explain some of the peculiarities in the system behavior.

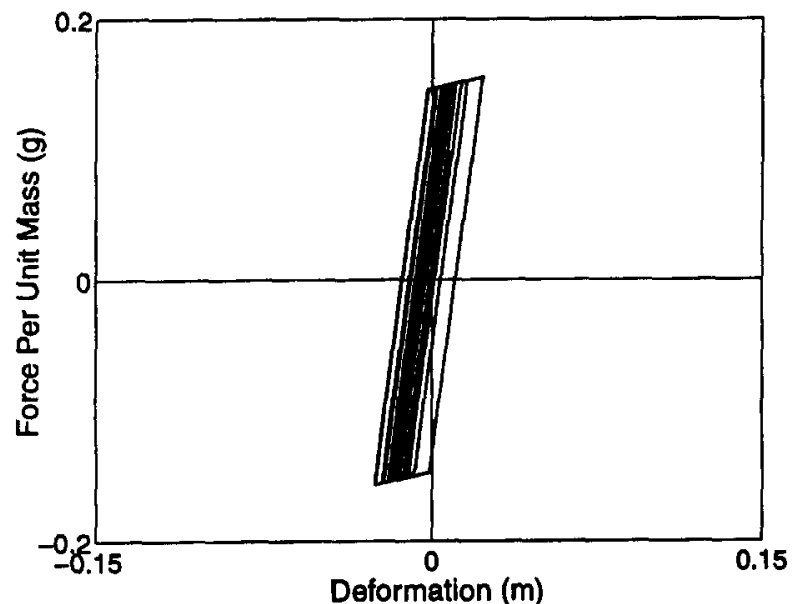

(a)

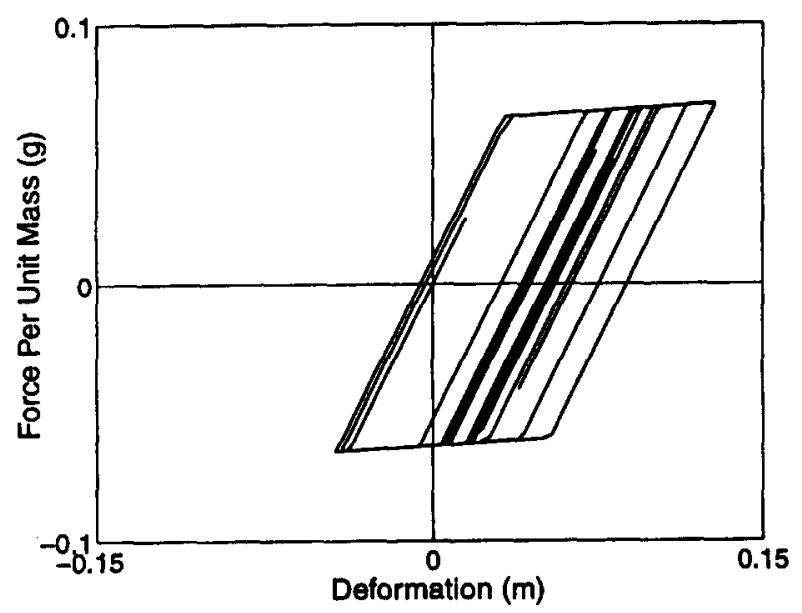

(c)
A slightly different definition of NHEDD was adopted by Chandler et al. (1996) wherein the constant one was ignored. Clearly, NHEDD computed according to the definition used in this investigation will be one plus the value computed by the definition adopted by Chandler et al. (1996). The trend for the ratio of NHEDD of a resisting element in asymmetric-plan system, $\mu_{H}$, and the corresponding symmetric-plan system, $\mu_{H O}$, used to characterize the effects of plan asymmetry is, however, more complicated. A comparison of the ratio computed according to the two definitions showed that the definition of Chandler et al. (1996) generally gives a larger value of the ratio (results are not presented for reasons of brevity).

\section{FORCE-DEFORMATION HISTORIES}

To gain insight into how plan asymmetry affects hysteretic energy dissipation demands on resisting elements, force-deformation histories of stiff- and flexible-side elements in the asymmetric-plan systems and the corresponding symmetricplan systems are compared in this section. The results were generated for UBC-94 designed systems with $T_{y}=0.5$ and 1 s; the former represents a short-period, low-rise building, whereas the latter corresponds to a midperiod, taller building. The presented results (Figs. 5 and 6) show the following trends.

The stiff-side element in the short-period, asymmetric-plan system undergoes inelastic cycles of smaller deformation magnitude and is required to dissipate less energy through hyster-

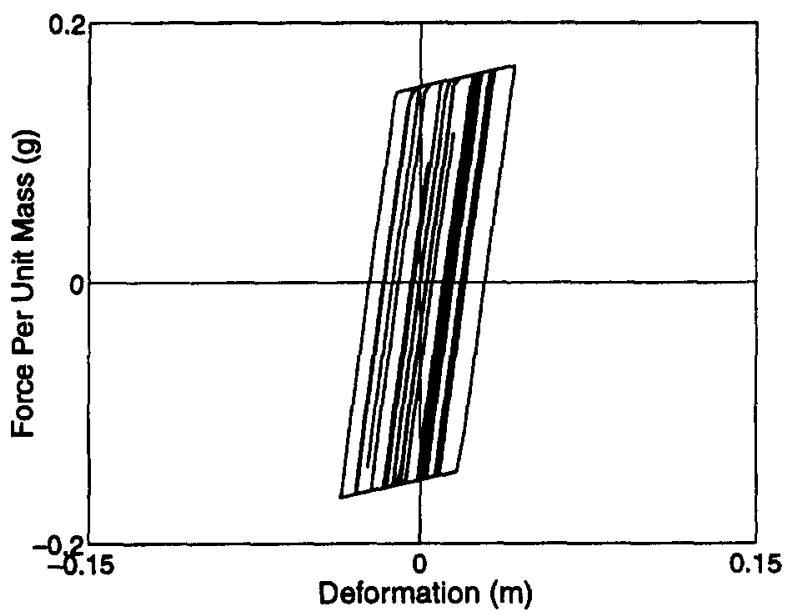

(b)

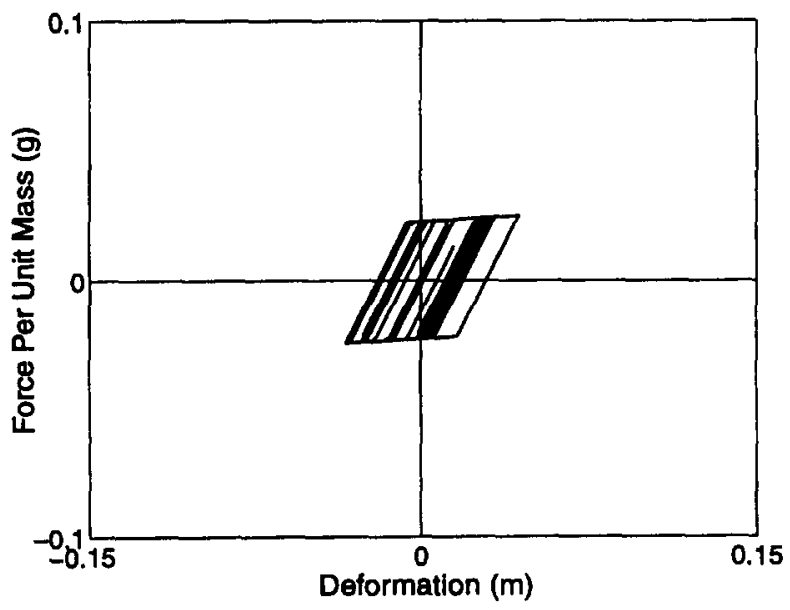

(d)

FIG. 5. Force-Deformation Historles of Resisting Elemente due to 1940 El Centro Earthquake: System Designed by UBC-94, $T_{y}=$ $0.5 s, \delta_{x y}=\delta_{s x}=0.3, \Omega_{0}=\Omega_{x}=\eta=1$, and $\gamma_{x}=0.5$ : (a) Stiff-Side Element, Asymmetric Syatem; (b) Stiff-Side Element, Symmetric Syatem; (c) Flexible-Side Element, Asymmetric System; (d) Flexible-Side Element, Asymmetrlc System 


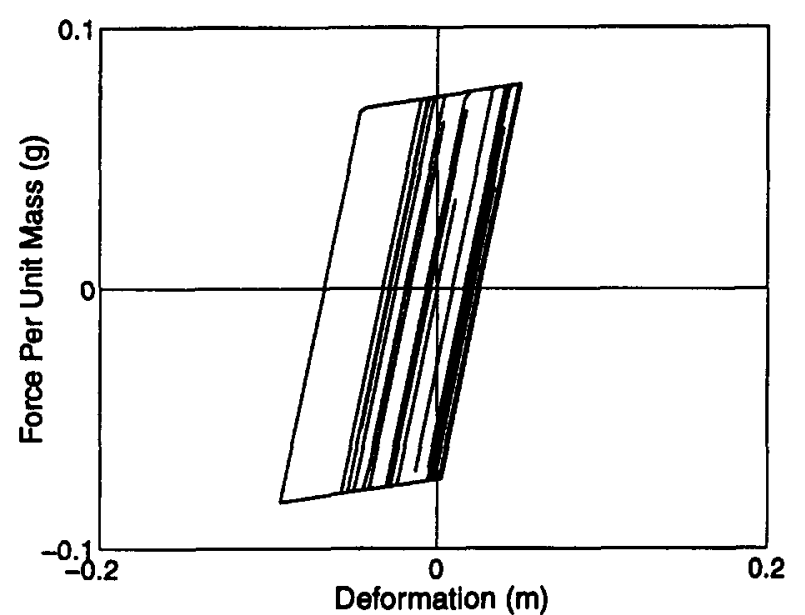

(a)

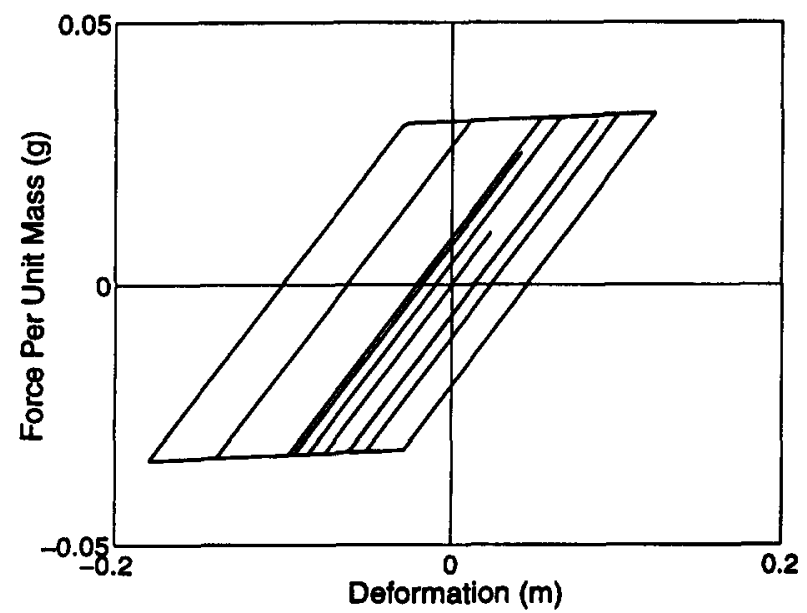

(c)

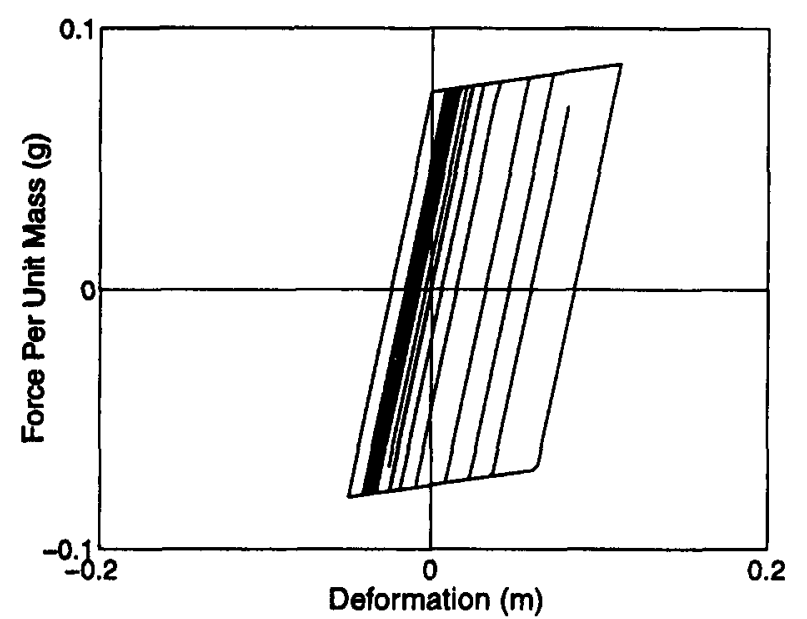

(b)

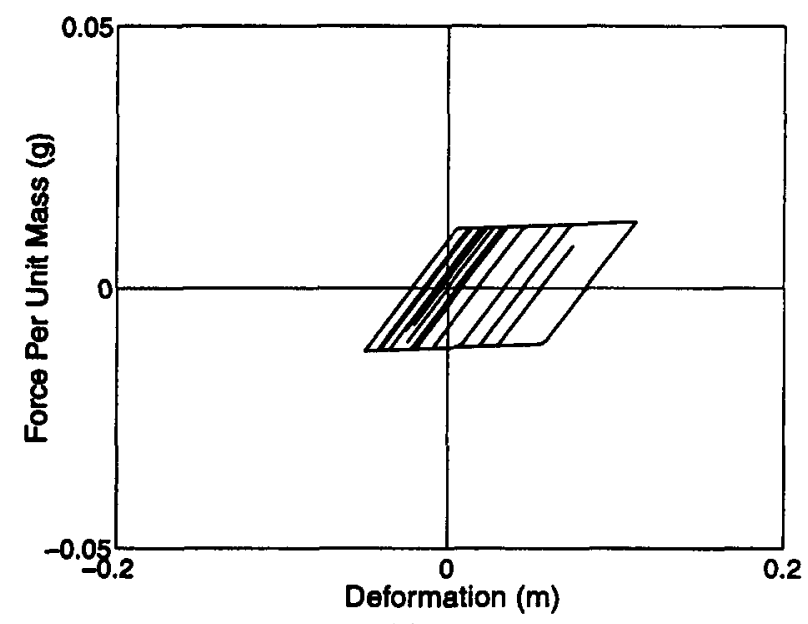

(d)

FIG. 6. Force-Deformation Histories of Resisting Elements due to 1940 El Centro Earthquake: System Designed by UBC-94, $T_{y}=1 s$, $\delta_{o y}=\delta_{o x}=0.3, \Omega_{0}=\Omega_{x}=\eta=1$, and $\gamma_{x}=0.5$ : (a) Stiff-Side Element, Asymmetric Syetem; (b) Stiff-Side Element, Symmetric System; (c) Flexible-Side Element, Asymmetric System; (d) Floxlble-Side Element, Asymmotric System

etic action, compared to the same element in the reference system [Figs. 5(a) and 5(b)]. The flexible-side element, on the other hand, experiences much larger inelastic cycles of both force and deformation magnitude [Figs. 5(c) and 5(d)]. The deformation of the largest inelastic cycle in the asymmetricplan system is more than twice that in the reference system; a similar observation was reported in previous investigations [e.g., Goel and Chopra (1990); Wong and Tso (1995)]. Obviously, the demand on a flexible-side element of a short-period asymmetric-plan system to dissipate energy through hysteretic action is significantly higher than on the same element in the symmetric-plan system. The inelastic cycles and energy demands in the stiff-side element of a midperiod asymmetricplan system are roughly of the same order of magnitude as those of the reference system [Figs. 6(a) and 6(b)]. The trends for the flexible-side element in the midperiod asymmetric-plan system are very similar to those in the short-period system, except that the difference between the energy dissipated in the two systems tends to be slightly smaller [Figs. 6(c) and 6(d)].

\section{ENERGY SPECTRA}

To evaluate how plan asymmetry affects the relative seismic input energy, energy dissipated by damping, and total energy dissipated by all resisting elements, spectra for these energy quantities of the asymmetric-plan systems are next compared with those of the reference system. For each system, the energy quantities used to generate these spectra were at a time equal to one beating cycle past the end of the earthquake. It was found that the instantaneous kinetic energy and strain energies in various resisting elements of a system were negligibly small at this time, indicating that the system, for all practical purposes, had stopped vibrating. Therefore, all the seismic energy input into the system had been dissipated by a combination of damping action and hysteretic action. In other words, the sum of the damping energy and the hysteretic energy was equal to the input energy.

The mean spectra for the three energy quantities are presented in Fig. 7 for systems designed according to UBC-94. These results show that the total input energy is about the same, whereas energy dissipated through damping is slightly larger in the asymmetric-plan system as compared to the reference system over the entire period range. The hysteretic energy, which is the difference between the energy input to the system and the energy dissipated through damping, is slightly smaller for the asymmetric-plan system. This is especially so for systems with a period longer than $0.4 \mathrm{~s}$.

The results shown in Fig. 7 provide an important clue to understanding the behavior of asymmetric-plan systems, that is, earthquakes do not necessarily impart more seismic energy or impose a higher total hysteretic energy dissipation demand on an asymmetric-plan system than on its symmetric counterpart. Therefore, the higher vulnerability of asymmetric-plan systems during earthquakes, evident either from data collected on building damage during actual earthquakes [e.g., Whittaker (1995); Esteva (1987)] or from analytical studies [e.g., 


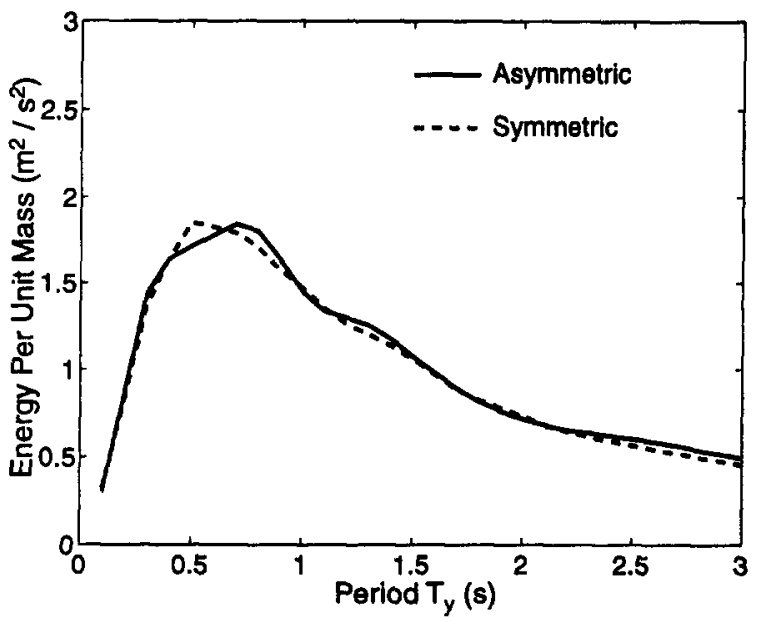

(a)

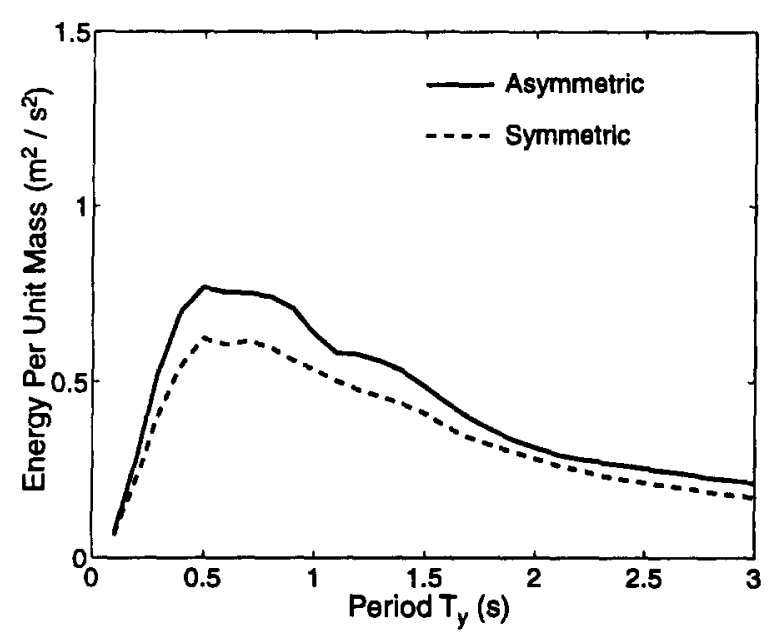

(b)

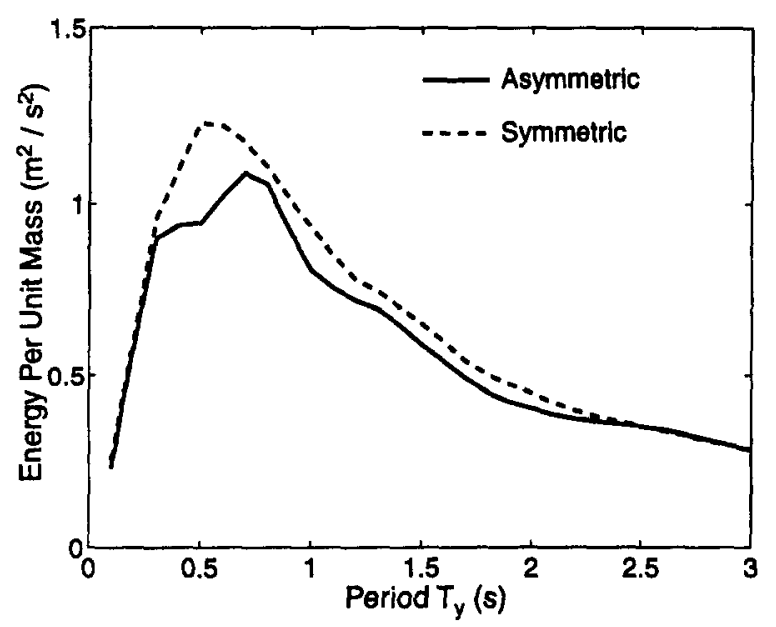

(c)

FIG. 7. Energy Spectra for Systems Designed by UBC-94: $\boldsymbol{\theta}_{\text {or }}$ $=\theta_{x x}=0.3, \Omega_{0}=\Omega_{x}=\eta=1$, and $\gamma_{x}=0.5$ : (a) Input Energy; (b) Damping Energy; (c) Total Hysteretic Energy

Goel and Chopra (1990)] appears to be related to how the total hysteretic energy is dissipated by various resisting elements. To further investigate this issue, the spectra of hysteretic energy was also generated for the individual elements and are presented in Fig. 8. These results lead to the following conclusions.

In the short-period range, the stiff-side element of an asymmetric-plan system experiences much smaller hysteretic en-

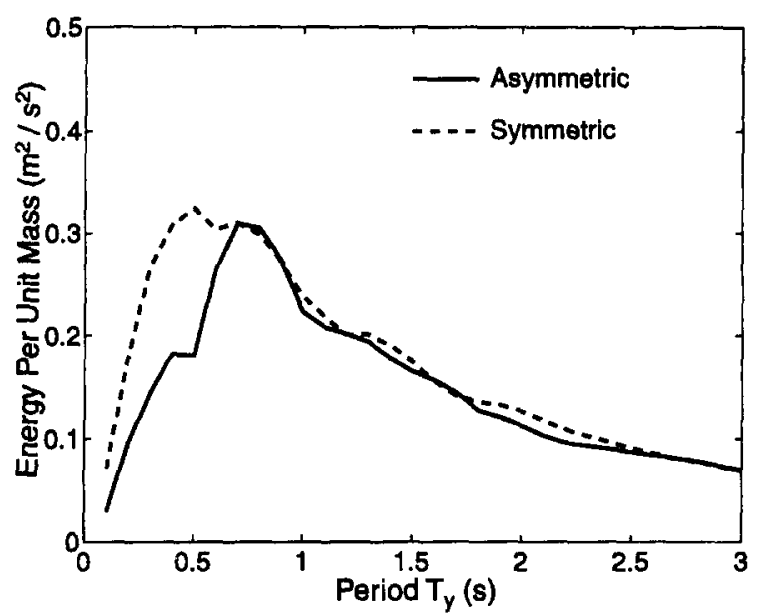

(a)

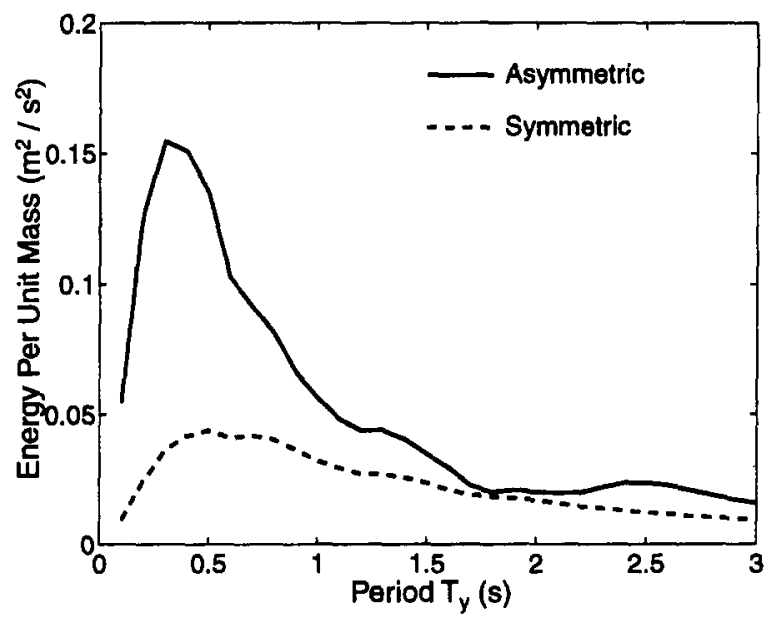

(b)

FIG. 8. Hysteretic Energy Spectra of Resisting Elements in Systems Designed by UBC-94: $\delta_{a y}=\delta_{e x}=0.3, \Omega_{0}=\Omega_{x}=\eta=1$, and $\gamma_{x}=0.5$ : (a) Stiff-Side Element; (b) Flexibie-Side Element

ergy demand than does the reference system [Fig. 8(a)]. In the midperiod range, however, the demands of the two systems are comparable. The demand on the flexible-side element of a symmetric-plan system is higher than on the symmetric-plan system for the entire period range, with the difference being particularly large in the short-period range [Fig. 8(b)]. Therefore, flexible-side elements in short-period, asymmetric-plan systems may be expected to experience significantly more damage and stiff-side elements no more damage compared to the same elements in the corresponding symmetric-plan system. Similar observations were also made by Correnza et al. (1995) based on ductility demands and by Chandler et al. (1996) based on hysteretic energy dissipation.

The results presented so far indicate that to prevent earthquake damage, flexible-side elements should possess larger hysteretic energy dissipation capacities (to meet higher demands) in an asymmetric-plan system than the same elements in the symmetric-plan system. It is well known that larger hysteretic energy dissipation capacity will result from: (1) capacity to withstand large deformations without failure, i.e., ductile (not brittle) behavior; (2) capacity to sustain a large number of load-reversal cycles without failure; and (3) stable force-deformation behavior (hysteresis loop) without significant pinching of the loop or degradation in stiffness and strength. Therefore, codes should provide detailing guidelines for the design of flexible-side elements in asymmetric-plan buildings that would ensure these noted qualities of member force-deformation behavior and, hence, enough energy dissi- 
pation capacity to meet the demand. Clearly these guidelines have to be more stringent for asymmetric-plan systems than for symmetric-plan systems.

\section{DUCTILITY DEMANDS}

\section{Peak Displacement DuctIllty Demand (PDDD)}

To assess the effects of plan asymmetry, the PDDD of a resisting element in the asymmetric-plan system, $\mu_{D}$, was normalized with the value in the same element of the reference system, $\mu_{D 0}$. A value of this ratio larger than one indicates that the PDDD was larger in the asymmetric-plan system as compared to the reference system. This ratio is presented in Fig. 9 for the stiff- and flexible-side elements of the system considered in this study.

It is apparent from these results that the stiff-side element in code-designed, short-period asymmetric-plan systems would not experience any larger inelastic demand than that in the same element of the reference systems [Fig. 9(a)]. However, for midperiod systems, the inelastic demand in the asymmetric-plan system may exceed that in the reference system. Among the three seismic codes considered in this investigation, excess demand tends to be the largest in systems designed by MFDC -87 and smallest in those designed by NBCC-95, with results for systems designed by UBC-94 falling between the two extreme cases. These trends are directly related to the strengths of the stiff-side element in systems designed accord-

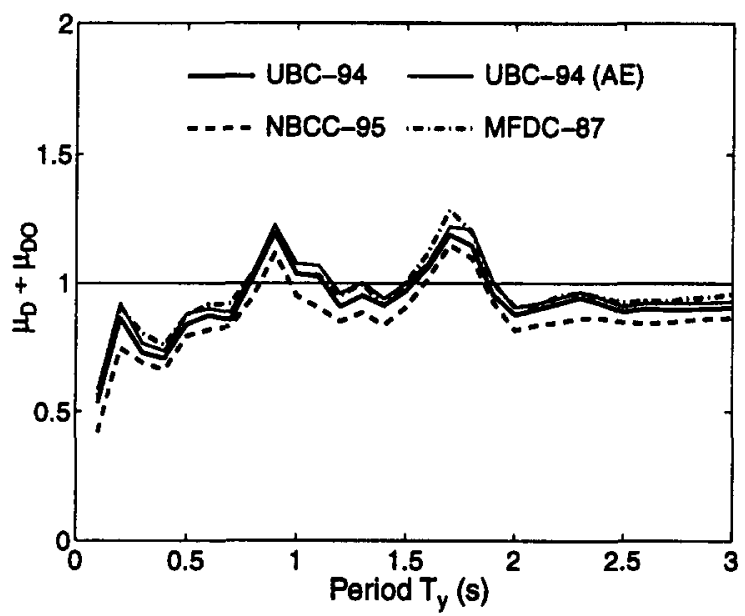

(a)

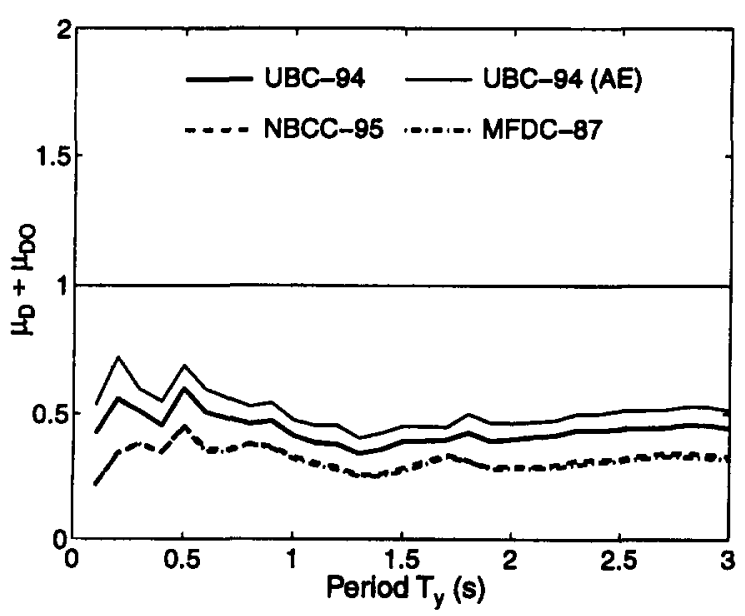

(b)

FIG. 9. Ratio of Peak Displacement Ductility Demands of Resisting Elements in Asymmetric-and Symmetric-Plan Systems: $\sigma_{x y}=\delta_{o x}=0.3, \Omega_{0}=\Omega_{x}=\eta=1$, and $\gamma_{x}=0.5$ : (a) Stiff-Side Element; (b) Flexible-side Element ing to the three codes [Fig. 2(a)]. The excess PDDD in stiffside elements of the system designed according to NBCC-95 was smallest because of the larger strength of this element. MFDC-87 led to the smallest strength of this element, and consequently the excess PDDD was the largest.

The flexible-side element in the asymmetric-plan system experiences PDDD much smaller than that in the reference system for all three codes [Fig. 9(b)]. Results are very similar for NBCC-95 and MFDC-87 due to similar strengths of this element resulting from these codes [Fig. 2(b)]. Furthermore, the PDDD in flexible-side elements of a system designed according to NBCC-95 and MFDC-87 is smaller than UBC-94 because of the larger strength resulting from the former codes.

\section{Normalized Hysteretic Energy Dissipation Demand (NHEDD)}

The NHEDD of a resisting element in the asymmetric-plan system, $\mu_{H}$, normalized with the value in the same element of the reference system, $\mu_{H O}$, was also computed and is presented in Fig. 10 for the stiff- and flexible-side elements of the system considered in this study. The trends for NHEDD in both elements are very similar to those based on PDDD except for minor differences. The differences are for the stiff-side element in midperiod systems. In particular, these systems experience much larger excess NHEDD compared to PDDD. These differences may be attributed to the fact that NHEDD captures

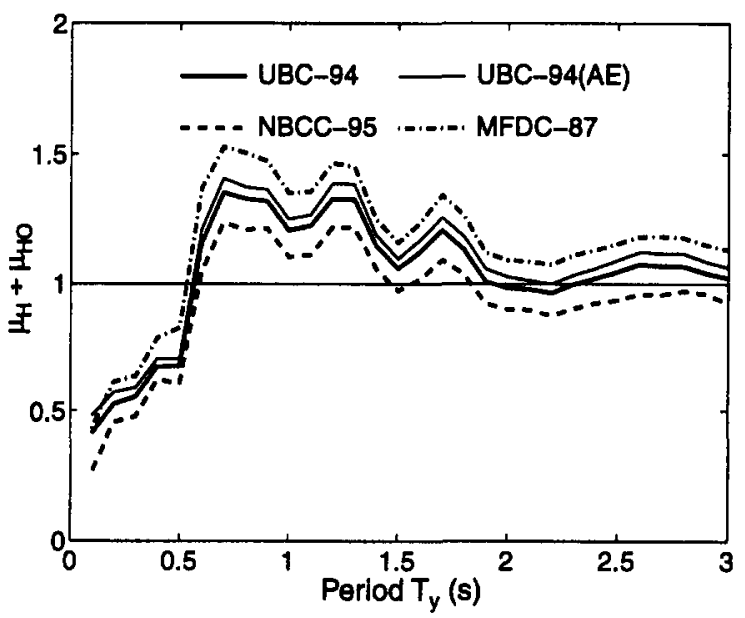

(a)

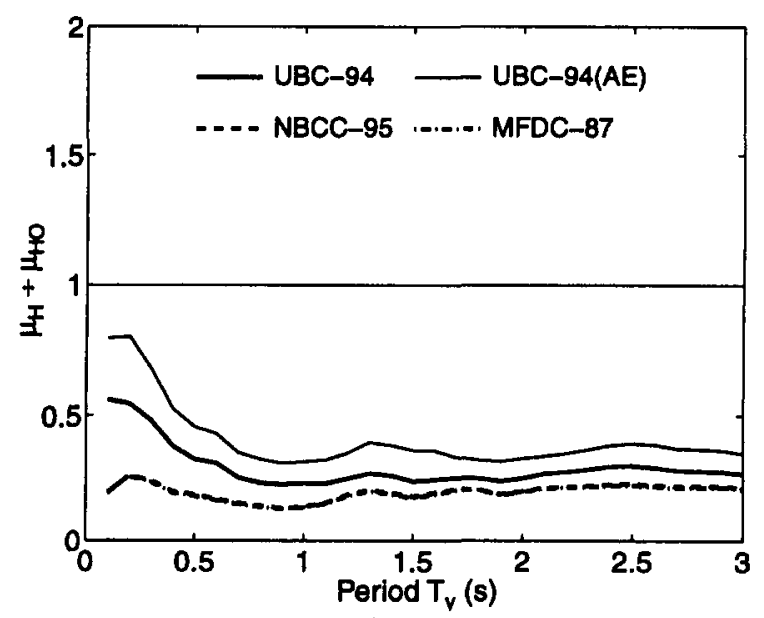

(b)

FIG. 10. Ratio of Peak Normalized Hysteretic Energy Ductility Demands of Resisting Elements In Asymmetric- and Symmetric-Plan Systems: $\delta_{a y}=\delta_{a x}=0.3, \Omega_{a}=\Omega_{x}=\eta=1$, and $\gamma_{x}=0.5:$ (a) Stiff-Side Element; (b) Flexible-Side Element 
the effects of cyclic load reversal on inelastic demand as opposed to PDDD, which represents inelastic demand only during the largest cycle.

\section{Comparison with Previous Investigations}

The trends observed in the preceding two sections are similar to those noted in several earlier studies that were based on monosymmetric systems subjected to only one directional earthquake [e.g., Chopra and Goel (1991); Tso and Zhu (1992)]. However, the excess ductility demand noted in this investigation is smaller compared to that in the previous investigations. This is primarily due to higher system and element strengths in this investigation resulting from the orthogonal effects.

The trend in this investigation about the ductility demands on the flexible-side element, however, differs significantly from that observed by Chandler et al. (1995) and Correnza et al. (1995) based on PDDD and by Chandler et al. (1996) based on NHEDD. These investigations found that significant additional ductility demands may arise in this element of shortand medium-period systems designed according to UBC-94. To further investigate this discrepancy, values of $\mu_{D} / \mu_{D 0}$ and $\mu_{H} / \mu_{H 0}$, with $\mu_{D 0}$ and $\mu_{H 0}$ computed for the reference system that was designed including the accidental eccentricity, were also computed. Such a reference system is similar to that used by Chandler et al. $(1995,1996)$ and by Correnza et al. (1995). The results were generated for systems designed according to UBC-94 and are included in Figs. 9 and 10, where they are denoted as UBC-94(AE). It is apparent that the curves for UBC-94(AE) are generally higher compared to those for UBC94, with the difference being much larger for the flexible-side element as compared to the stiff-side element. But the curves for the flexible-side element remain below one for the entire range of period values, indicating that no excess ductility demand occurs on this element-a conclusion that is still in disagreement with that of Chandler et al. (1995, 1996) and Correnza et al. (1995). One plausible explanation is that the much lower system and element strengths of one-directional systems used in these investigations, compared to the twodirectional system of the present study. The higher values of NHEDD observed by Chandler et al. (1996) are also due to the different definitions of NHEDD, as noted previously.

\section{INTERPRETATION OF RESULTS}

The results presented so far indicate that hysteretic-energy demands on the flexible-side elements of code-designed asymmetric-plan buildings are significantly higher than on the same elements of the corresponding symmetric-plan buildings. If these elements are not designed to accommodate the higher demands, they may fail, which in turn may lead to structural collapse during earthquakes. Failure in many street corner buildings during the 1985 Mexico and 1995 Kobe earthquakes appears to be due to such a lack of hysteretic-energy dissipation capacity. Many of these buildings were designed and constructed prior to the development of modern seismic codes and included brittle lateral-load resisting elements such as masonry infilled frames and nonductile reinforced-concrete frames (Esteva 1987; Whittaker 1995). As mentioned previously, such elements cannot be expected to dissipate much hysteretic energy due to limited deformation capacity (i.e., premature brittle failure) and rapid degradation in strength and stiffness under cyclic loading during earthquakes.

Vulnerability of street corner buildings during earthquakes cannot be explained based on ductility demands (NHEDD or PDDD). The previously presented results showed that ductility demands on the flexible-side element in code-designed asymmetric-plan systems are not necessarily higher than the same element in the corresponding symmetric-plan systems. Therefore, these results, contrary to the results for hysteretic-energy demands, indicate no vulnerability of the flexible-side element. Such inability of NHEDD or PDDD in predicting damage patterns in street corner buildings is related to the infinite deformation capacity and, hence, hysteretic-energy dissipation capacity, assumed in this and most other investigations. An accurate assessment of the damage potential in street corner buildings that failed during the 1985 Mexico and 1995 Kobe earthquakes can only be obtained by incorporating appropriate force-deformation relationships for nonductile lateral-load resisting elements; investigation along these lines is planned in the future. If these buildings had been designed to accommodate the additional hysteretic-energy dissipation demand, both the hysteretic-energy demand results and the ductility demands (NHEDD or PDDD) would lead to the same conclusion-i.e., flexible-side elements are not necessarily more vulnerable in asymmetric-plan systems as compared to the same elements in the corresponding symmetric-plan systems.

\section{CONCLUSIONS}

This investigation on inelastic seismic responses of codedesigned, asymmetric-plan systems with large eccentricities in both directions and subjected to two components of ground motion has led to the following conclusions.

The total energy input to the system is about the same, whereas the total hysteretic energy dissipated by all elements is slightly smaller for the asymmetric-plan system as compared to the corresponding symmetric-plan system.

The flexible-side elements undergo much larger hysteretic energy demands in an asymmetric-plan system than in the corresponding symmetric-plan systems. The stiff-side elements, on the other hand, do not necessarily experience any larger hysteretic demands in asymmetric-plan systems.

The stiff-side element may experience larger ductility demands, PDDD as well as NHEDD, in midperiod, asymmetricplan systems when compared to the same element in the corresponding symmetric-plan systems. The flexible-side element, on the other hand, undergoes much smaller ductility demands in asymmetric-plan systems.

The flexible-side elements in asymmetric-plan buildings with large eccentricities in both directions may be more vulnerable to earthquakes if not designed to accommodate the higher hysteretic-energy demands imposed by earthquakes. Although this observation is strictly valid only for the types of systems considered in this investigation, it can be extended to explain failure in many street corner buildings during the 1985 Mexico and 1995 Kobe earthquakes, as these buildings contained lateral-load resisting elements with limited hystereticenergy dissipation capacity, i.e., nonductile, brittle elements.

Building codes should provide detailing guidelines for asymmetric-plan buildings that would ensure enough energy dissipation capacity to meet the demand. These guidelines should be more stringent for asymmetric-plan systems than for symmetric-plan systems.

This investigation is based on the inelastic responses of systems to five sets of ground motions recorded at rock sites in California. These motions were selected because of similar elastic response spectra. It would be useful to examine the validity of these results for ground motions for other soil conditions and locations and for ground motions with similar energy spectra. It would also be useful to further examine how increasing the strength of a lateral-load resisting element, as required by code torsional provisions, affects its stiffness and various ductility demands. 


\section{APPENDIX. REFERENCES}

Chandler, A. M., Correnza, J. C., and Hutchinson, G. L. (1995). "Influence of accidental eccentricity on inelastic seismic torsional effects in buildings." Engrg. Struct., 17(7), 167-178.

Chandler, A. M., Correnza, J. C., and Hutchinson, G. J. (1996). "Seismic torsional provisions: Influence on element energy dissipation." $J$. Struct. Engrg., ASCE, 122(5), 494-500.

Chopra, A. K., and Goel, R. K. (1991). "Evaluation of torsional provisions in seismic codes." J. Struct. Engrg., ASCE, 117(12), 3762-3782.

Correnza, J. C., Hutchinson, G.L., and Chandler, A. M. (1992). "A review of reference models for assessing inelastic seismic torsional effects in buildings." Soil Dyn. and Earthquake Engrg., 11(8), 465-484.

Correnza, J. C., Hutchinson, G. L., and Chandler, A. M. (1995). "Seismic response of flexible-edge elements in code-designed torsionally unbalanced structures." Engrg. Struct., 17(3), 158-166.

De Stefano, M., Faella, G., and Ramasco, R. (1993). "Inelastic response of code-designed asymmetric systems." Eur. Earthquake Engrg., 7(3), 3-17.

Esteva, L. (1987). "Earthquake engineering research and practice in Mexico after the 1985 earthquakes." Bull. of the New Zealand Nat. Soc. for Earthquake Engrg., 20(3), 159-200.

Goel, R. K., and Chopra, A. K. (1990). "Inelastic seismic response of one-story, asymmetric-plan systems." Rep. No. UCB/EERC-90/14, Earthquake Engineering Research Center, University of California, Berkeley, Calif.

Goel, R. K., and Chopra, A. K. (1994). "Dual-level approach for seismic design of asymmetric-plan buildings." J. Struct. Engrg., ASCE, 120(1), $161-179$.

Gomez, R., and Garcia-Ranz, F. (1988). "The Mexican earthquake of September 19, 1985-Complementary technical norms for earthquake resistant design." Earthquake Spectra, 4(3), 441-460.

Mahin, S. A., and Bertero, V. V. (1981). "An evaluation of inelastic seismic design spectra," J. Struct. Div., ASCE, 107(9), 1777-1795.

National building code of Canada. (1995). National Research Council, Ottawa, Canada.

Rutenberg, A. (1992). "Nonlinear response of asymmetric building structures and seismic codes: A state of the art review." Eur. Earthquake Engrg., 6(2), 3-19.

Tso, W. K., and Zhu, T. J. (1992). "Design of torsionally unbalanced structural systems based on code provisions. I: Ductility demand." Earthquake Engrg. and Struct. Dyn., 21(7), 609-627.

Uang, C.-M., and Bertero, V. V. (1990). "Evaluation of seismic energy in structures." Earthquake Engrg. and Struct. Dyn., 19(1), 77-90.

Uniform building code. (1994). International Conference of Building Officials, Whittier, Calif.

Whittaker, S. (1995). "NSF-EERC Kobe earthquake reconnaissance report." Draft Chapter on Engineered Buildings, Earthquake Engineering Research Center, University of California, Berkeley, Calif.

Wilson, E. L., Suharwardy, I., and Habibullah, A. (1995). "A clarification of the orthogonal effects in a three-dimensional seismic analysis." Earthquake Spectra, 11(4), 659-666.

Wong, C. M., and Tso, W. K. (1995). "Evaluation of seismic torsional provisions in uniform building code." $J$. Struct. Engrg., ASCE, $121(10), 1436-1442$. 